\title{
Population screening for glaucoma in UK: current recommendations and future directions
}

\author{
Sana Hamid (D) ${ }^{1}$, Parul Desai (iD) ${ }^{1}$, Pirro Hysi (D) ${ }^{2,3}$, Jennifer M. Burr ${ }^{4}$ and Anthony P. Khawaja (D) ${ }^{\circledR}$ \\ (c) The Author(s) 2021
}

\begin{abstract}
Effective population screening for glaucoma would enable earlier diagnosis and prevention of irreversible vision loss. The UK National Screening Committee (NSC) recently published a review that examined the viability, effectiveness and appropriateness of a population-based screening programme for primary open-angle glaucoma (POAG). In our article, we summarise the results of the review and discuss some future directions that may enable effective population screening for glaucoma in the future. Two key questions were addressed by the UK NSC review; is there a valid, accurate screening test for POAG, and does evidence exist that screening reduces morbidity from POAG compared with standard care. Six new studies were identified since the previous 2015 review. The review concluded that screening for glaucoma in adults is not recommended because there is no clear evidence for a sufficiently accurate screening test or for better outcomes with screening compared to current care. The next UK NSC review is due to be conducted in 2023. One challenge for POAG screening is that the relatively low disease prevalence results in too many falsepositive referrals, even with an accurate test. In the future, targeted screening of a population subset with a higher prevalence of glaucoma may be effective. Recent developments in POAG polygenic risk prediction and deep learning image analysis offer potential avenues to identifying glaucoma-enriched sub-populations. Until such time, opportunistic case finding through General Ophthalmic Services remains the primary route for identification of glaucoma in the UK and greater public awareness of the service would be of benefit.
\end{abstract}

Eye (2022) 36:504-509; https://doi.org/10.1038/s41433-021-01687-8

\section{INTRODUCTION}

Glaucoma is the second most common cause of blindness globally [1]. Unlike cataract, the leading cause of blindness, glaucoma causes irreparable vision loss. This, in combination with the progressive nature of the disease, means that early detection and treatment are critical for preventing blindness from glaucoma [2]. The asymptomatic nature of mild glaucoma means that examination is required for early detection [2]. While the necessity for early diagnosis to prevent blindness may suggest that glaucoma is a good candidate for population screening, inadequate tests for the relatively low prevalence in the population have so far precluded a national screening programme. Currently in the UK, glaucoma detection is opportunistic, most frequently by optometrist assessment in the community. A recent population-based study in Northern Ireland suggests that the majority of people with glaucoma are undetected or are at least unaware of their diagnosis [3].

The UK National Screening Committee (UK NSC) regularly reviews the evidence supporting population screening to provide recommendations to the government and National Health Service (NHS) in the UK. In 2019, the UK NSC updated their 2015 review of available evidence supporting population screening for the commonest form of glaucoma, primary open-angle glaucoma (POAG) [4]. The 2019 report states that the UK NSC still cannot recommend population screening for POAG in adults due to inadequate supporting evidence. In this article, we present a summary of the findings of the 2019 UK NSC review and discuss potential future directions that may enable effective population screening of POAG.

\section{UK NATIONAL SCREENING COMMITTEE EXTERNAL REVIEW OF SCREENING FOR GLAUCOMA, 2019 \\ Aims}

The viability, effectiveness and appropriateness of a populationbased screening programme for POAG were assessed by the UK National Screening Committee (UK NSC) and their review was published in December 2019 [4]. Two key questions relating to the UK NSC screening criteria were evaluated to determine whether there was any new relevant evidence published since the last review in March 2015 and therefore whether to reconsider the recommendation of the last review against population screening for POAG in the UK. These two questions considered firstly whether there is a valid, accurate screening test for POAG and secondly whether evidence exists that POAG screening reduces morbidity from the condition compared with standard diagnosis and care. The NSC commissioned the evidence review which was carried out using rapid review methodologies [5].

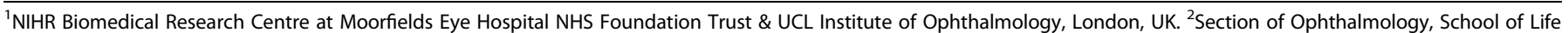

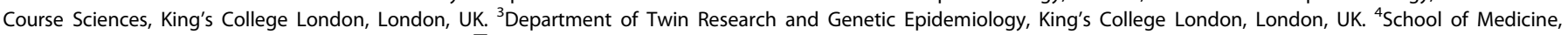
University of St Andrews, St Andrews, Scotland, UK. ${ }^{凶}$ email: anthony.khawaja@ucl.ac.uk 


\section{Methods}

A systematic search of three databases (Medline, Embase and Cochrane) was undertaken to identify relevant studies published since 1 October 2014 up to 25 March 2019 in relation to the key questions; search criteria are summarised in Table 1.

\section{Findings: is there a good screening test for POAG?}

The review initially searched for information on the diagnostic accuracy of screening tests for POAG in the adult population to assess whether there is a simple, safe, precise, and validated screening test. When this question was assessed by the UK NSC review in 2015, one meta-analysis, one systematic review, six studies assessing functional tests and two studies assessing structural tests were analysed. Overall, the studies had small sample sizes and a wide variability in the sensitivity and specificity of the available tests were reported, deeming them unsuitable for use in population screening.

In the 2019 review, six new studies [6-11] met the inclusion criteria after full-text review (Tables 2 and 3). The studies reported POAG screening test performance results in populations with unknown ocular history; sample sizes ranged from 220 [9] to 4167 [7]. Five of the studies targeted people with a higher risk of developing POAG due to ethnicity, age, or family history [6, 8-11]. All studies used combinations of functional and structural types of screening test and employed a screening algorithm or model to determine who should be referred for a definitive eye examination (the reference standard). The results of the definitive eye examination were typically 'no glaucoma', 'suspected glaucoma' or 'definitive glaucoma'. The studies combined the tests used at the screening examination to calculate screening performance statistics. No studies combined the same screening tests with the same cut-offs. The reported performance of individual and combined screening tests from these studies are summarised in Tables 2 and 3, respectively.

There was no agreement about the most effective combination of tests or cut-off levels that should be used in a screening examination for POAG. The screening test performance statistics reported were variable and not comparable across studies. The review concluded that there is an insufficient evidence base for a simple, safe, precise and validated screening test with known distribution of test values and agreed suitable cut-off levels.

\section{Findings: does screening for POAG reduce morbidity?}

The review subsequently searched for studies that investigated whether a screening programme for POAG was effective in reducing the morbidity associated with the condition. The March 2015 review assessed four studies [12-15], all of which concluded that there was insufficient evidence to recommend populationbased screening for POAG. Burr et al. also reported that glaucoma screening of a population selected on age is unlikely to be costeffective and that there is uncertainty surrounding test performance as well as around engagement with a POAG screening programme [15]. The 2013 US Preventative Services Task Force recommendation statement reported concerns about overdiagnosis and possible overtreatment as not all people go on to develop visual impairment [14]. Ervin et al. did not identify any studies to provide evidence for links between whether glaucoma screening impacted on visual field loss, visual impairment, optic nerve damage, intraocular pressure or patient-reported outcomes [13].

In the 2019 review, three additional studies reporting results of screening programmes were identified $[8,11,16]$. However, none of the studies reported any data regarding treatment outcomes or overall outcomes of the whole-screening programme performance. Anton et al. performed a cross-sectional study and reported detection rates $(4.1 \%$ of those screened had glaucoma or suspect glaucoma) and costs of a screening programme (1410 $€$ per case detected) using tonometry and imaging devices in at-risk
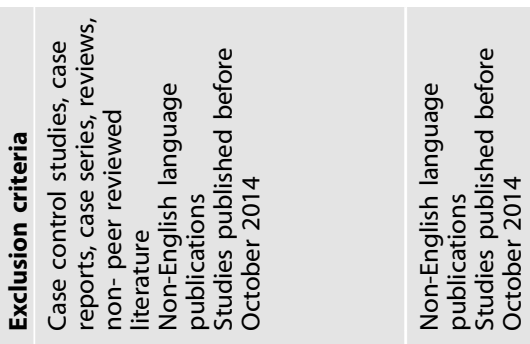
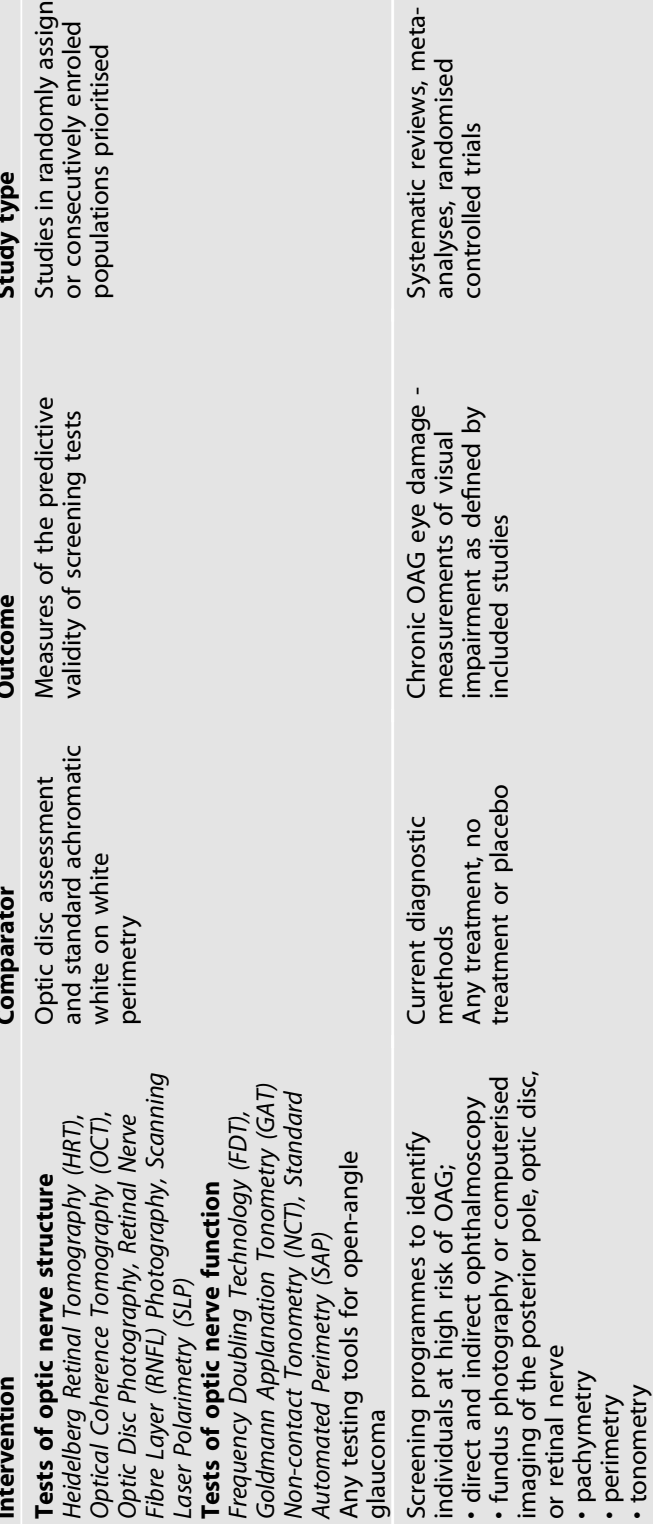

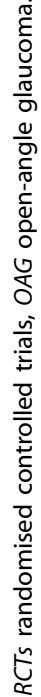


population in Spain [16]. The screening performance statistics were beyond the scope of the study and the treatment outcomes and overall screening programme performance were not reported. A study by Hark et al. examined the agreement of ocular findings between telemedicine eye screening comprising fundus photography, tonometry, and clinical information, with diagnosis made from a comprehensive eye examination in an at risk population in Philadelphia [11]. They reported $29.2 \%$ detection rate of glaucoma-related eye disease (glaucoma, glaucoma suspect and narrow angle) in those screened; the diagnosis confirmation rate was $80 \%$ after eye examination. Performance statistics were not reported. Zhao et al. focussed on the accuracy of screening tests in the development of a screening programme in an at-risk population in Baltimore, but did not report performance statistics [8].

The UK NSC review did not identify any randomised controlled trials examining the effectiveness of a POAG screening programme in reducing disease morbidity from the condition compared with usual diagnosis and care.

\section{Conclusion and recommendations}

Following this assessment, the committee concluded that none of the existing screening protocols should be recommended for POAG in adults. This is in line with the previous UK NSC recommendation from 2015. It is also in agreement with the 2017 National Institute for Health and Care Excellence (NICE) glaucoma guideline [17] which assessed the accuracy of five risk tools to predict conversion to POAG in people with ocular hypertension; the report concluded that the current evidence on the sensitivity and specificity of risk tools for developing POAG is of moderate to low quality, with all studies having a high or very high risk of bias [17]. The next UK NSC review is due to be conducted in 2022/23.

\section{Limitations}

Although the evidence review underpinning the UK NSC recommendation has potential minor limitations such as searching only three electronic databases and not the grey literature, the main limitation is insufficient evidence to judge whether population screening for glaucoma is worthwhile in terms of a suitable screening test and whether the benefits of a glaucoma screening programme outweigh any potential harms. Ideally, before instigating any screening programme evidence of effectiveness from a randomised controlled screening trial is required. However, conducting such as trial of glaucoma screening is unlikely to be the best use of research resources [15] until some fundamental questions regarding what any future glaucoma screening programme is trying to achieve are addressed. In particular, the target population, the type and severity of glaucoma one is trying to detect and underlying consensus definitions.

\section{FUTURE DIRECTIONS}

One of the challenges to effective population screening for glaucoma is the relatively low disease prevalence in the general population combined with the reasonable performance of opportunistic case finding. A low disease prevalence results in a poor positive predictive value of even very accurate tests (see below). While screening for glaucoma in younger compared to older people is more likely to be beneficial in terms of reducing glaucoma disability, the prevalence of POAG in the general population selected on age alone, even in older age cohorts, is too low for a population-based screening programme to be recommended. Such a programme would overburden health services. For example, if we assume the prevalence of glaucoma in an inception cohort aged 50 years to be $0.9 \%$ [18], and the performance of the screening test or programme to be $73 \%$ 
Table 3. Combined screening test performance for suspected and definitive POAG.

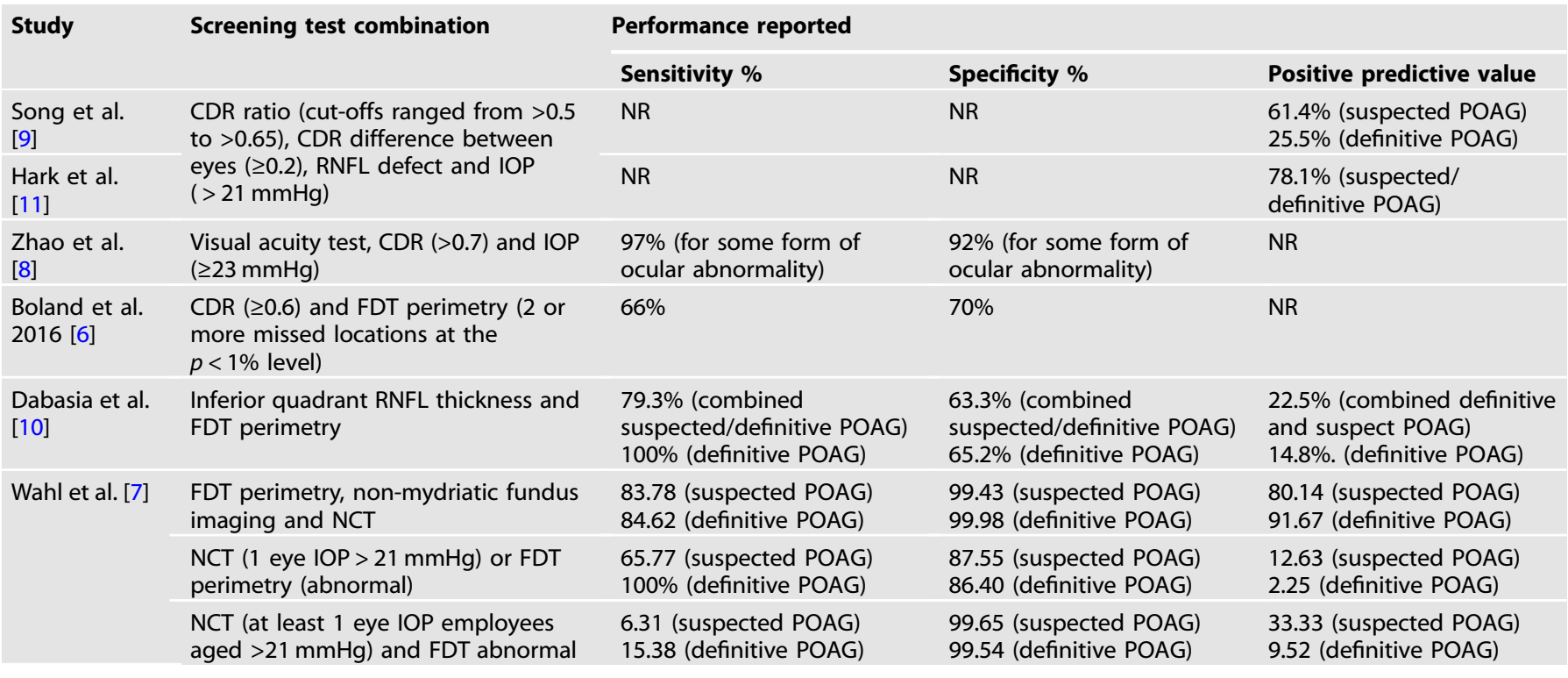

$C D R$ cup-disc ratio, FDT frequency doubling technology, NR not reported, NCT non-contact tonometry, IOP intraocular pressure, GCC ganglion cell complex, $R N F L$ retinal nerve fibre layer.

sensitivity and $96 \%$ specificity [19], the positive predictive value will be relatively poor (Fig. 1). In this scenario, a positive test that will result in referral will be a false positive $86 \%$ of the time. In other words, more than 8 out of 10 referrals will be unnecessary, generating wasteful burden on secondary care services. Overburdened secondary eye care services have been highlighted as a major problem and a cause of delays to the care of high-risk patients $[20,21]$. Therefore, an efficient screening programme is required to have a higher positive predictive value (i.e., lower false positive referral rate). This may be achieved either by targeted testing of higher risk subgroups rather than inviting people selected on age alone. Modelling suggests that initiating a screening programme for a high-risk subset of a cohort aged 50 years, with an expected prevalence of POAG of around $4-5 \%$ (rather than the $0.9 \%$ in the general population aged 50 years), might be worthwhile $[15,18]$. However, the systematic identification of higher glaucoma risk subgroups in the population is challenging.

One strategy could be targeting screening to families of people with POAG. First-degree relatives of glaucoma patients have been shown to have a ninefold increased risk of developing glaucoma in their lifetime compared to relatives of controls in the population-based Rotterdam Study [22]. The higher prevalence of glaucoma in first-degree relatives will improve the positive predictive value of any test, thereby reducing false-positive referrals. While a formal screening programme targeting firstdegree relatives of glaucoma sufferers makes theoretical sense, the practical application may not be straightforward. For example, there would need to be a clear definition of which glaucoma patients should have their relatives screened (e.g., POAG only or other types of glaucoma as well) and these individuals would need to be accurately identified at scale nationally. Self-report of disease status is unreliable for glaucoma overall, and would likely be worse for specific sub-types of glaucoma. In the future, increasing uptake of electronic medical records may enable a digital national glaucoma registry which could inform targeted screening of first-degree relatives. However, many challenges would persist including the practicality and ethical and information governance implications of sharing health information or linkage of health records with relatives.

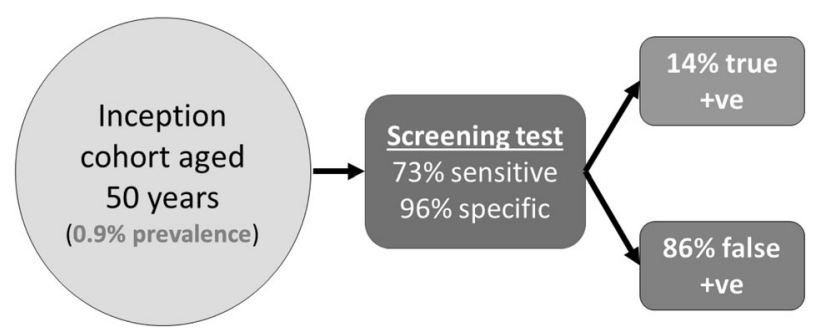

Fig. 1 Predictive performance of a screening test (73\% sensitivity and $96 \%$ specificity) when applied to an inception cohort of 50 years of age with a glaucoma prevalence of $0.9 \%$.

In recent years, there has been great progress in the discovery of the genetic determinants of POAG [23]. Over 100 common genetic variants have been identified which each contribute a small increased risk of high intraocular pressure (IOP) or POAG [24]. When combined together, these variants cumulatively can predict who will develop POAG with an area under the ROC curve of $76 \%$ [24]. Further adding genetic variants which are associated with vertical cup-disc ratio and glaucoma and creating a polygenic risk score (PRS) for POAG has also demonstrated potential for identifying individuals in a population who are at high risk for disease [25]. In the Australia and New Zealand Registry of Advanced Glaucoma (comprising 3071 advanced POAG cases and 6750 historic controls of European descent), participants in the top decile of PRS were at a 15-fold increased risk of developing advanced glaucoma compared to the bottom decile [25]. Compared to the remaining $90 \%$ of the cohort, participants in the top decile of PRS were at a 4.2-fold increased risk of advanced glaucoma.

If genetic data were available for the general population, we would be able to target a glaucoma screening programme to individuals at the highest genetic risk of developing advanced glaucoma. If we apply the 4.2 -fold increased risk in the top $10 \%$ to the 50-year-old inception cohort, we would predict a glaucoma risk of around $4 \%$. If we were then to target screening to this enriched sub-population with the aforementioned screening test (73\% sensitivity and $96 \%$ specificity), the false-positive rate would 


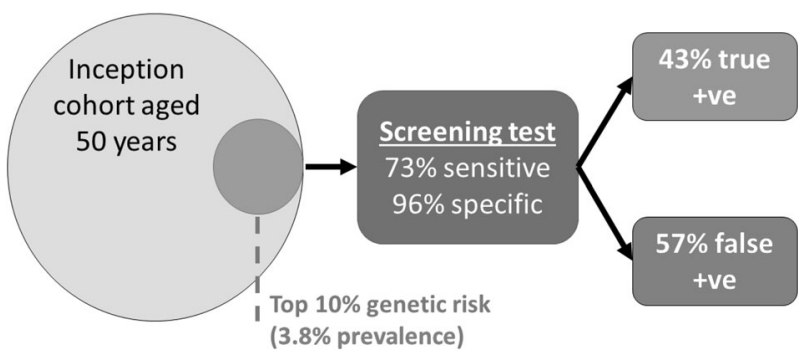

Fig. 2 Predictive performance of a screening test (73\% sensitivity and $96 \%$ specificity) targeted to a subset of the inception cohort aged 50 years with high genetic risk for glaucoma (an enriched prevalence of $0.9 \% \times 4.2=3.8 \%$ ).

fall substantially (Fig. 2). For each positive screening test result, there will be a $57 \%$ chance of a false positive. This example highlights the potential gains of targeting high-risk sub-populations, and the significant role genetic testing could play. While genetic testing is not routine in the general population currently, its affordability and applicability for multiple diseases make it a likely possibility in the future. Targeted glaucoma screening of people at high genetic risk of glaucoma is currently being examined prospectively in a large Dutch cohort [26], which if successful, could provide strong support for genotype-based targeting screening for glaucoma in the future. It will be necessary to determine the optimal testing strategy for people identified to be at high risk (e.g., top decile of genetic risk and aged 50); this may be community screening every 2 years until a glaucoma diagnosis or until an upper age-limit when it is deemed unlikely to develop vision loss due to glaucoma during remaining life. It will also be necessary to demonstrate that genetic testing is feasible and acceptable to the public, and to compare the innovative screening strategy with current case detection in a prospective randomised trial. Another major challenge is ensuring that any prediction model is generalisable to diverse populations and people of different ethnicities. To date, the majority of genomewide association studies have examined people of European descent. While there appears to be good generalisability of genetic loci between European and Asian populations, there may be less overlap and correlation between European and African populations $[27,28]$. Future work in this field must aim to improve genetic discovery in non-European ethnic groups to enable the development and validation of prediction tools that can be deployed equitably in the future.

Another potential tool to help enable effective population screening for glaucoma is artificial intelligence-assisted image interpretation. Deep learning techniques have enabled algorithms that can classify optic disc images according to glaucoma risk and the need for further examination for glaucoma [29]. In some cases, the algorithm has been trained on images graded by multiple experts, with the resultant algorithm outperforming any single glaucoma expert in independent test sets [30]. While it may be unlikely for the positive predictive value of such an algorithm to be adequate when applied to the general population, it may be that the algorithm is used to identify a subset of the population that should be screened (rather than immediate referral). Similar to Fig. 2, screening a sub-population enriched for glaucoma (as detected by an optic disc image deep learning algorithm) may be effective due to reduced false positives compared to screening the general population. In addition, multimodal algorithms incorporating data and images on visual fields, IOP and optical coherence tomography (OCT) may reach the point of adequate predictive ability, even in a general population. Ultimately, approaches that combine both genetic prediction and deep learning algorithms may be developed.
Until effective population screening or targeted screening programmes are achievable, opportunistic case finding through General Ophthalmic Services (GOS) will remain the primary route for identification of both symptomatic and asymptomatic glaucoma in the UK. The GOS (commonly referred to as the "sight test") is provided by optometrists in community optical practice, but with distinct differences in the contract across each of the UK nations. It is provided as an NHS service for those meeting a range of eligibility criteria; criteria pertinent to glaucoma are as follows: having a diagnosis of glaucoma, aged 40 or over and either a parent, sibling or child that has been diagnosed with glaucoma, advised by an ophthalmologist as being at risk of glaucoma, aged 60 years and over [31]. The exception for this being Scotland where a sight test is provided as a universal NHS service to the whole population [32]. In addition, the contracting arrangements in all the devolved nations allow for the provision of supplementary services to improve the quality of decision-making for onward referral to specialist ophthalmic care [32-34], which include repeat measures and referral refinement recommended by NICE [17]. In England, the commissioning of these supplementary services are encouraged and will be central to the models of care for glaucoma for the Integrated Care Systems that are currently being established $[35,36]$. Despite these national differences, the GOS has an established process and defined clinical and professional standards. Greater public awareness of the service would not only improve health literacy facilitating healthier choices, but also improve case finding for a range of eye conditions including glaucoma, in the population at risk.

It should be noted that the clinical and cost-effectiveness of glaucoma screening depends on multiple factors that are specific to the healthcare setting. Modelling studies in Finland [37] and China [38] have suggested that population screening for glaucoma may be cost-effective in those healthcare settings. However, it is acknowledged that such models have uncertainty and can be sensitive to the specificity of diagnostic tests and cost of screening [37]. Another factor that is difficult to predict and can influence the effectiveness of a screening programme is the attendance rate.

Glaucoma remains an important cause of avoidable sight loss in England and Wales [39]. Effective population screening would enable earlier diagnosis and prevention of irreversible vision loss. Major advances in our ability to predict glaucoma risk using genetic markers, increasingly affordable genotyping, and advances in machine learning techniques all provide promise to enable innovative solutions for effective glaucoma screening in the future.

\section{REFERENCES}

1. Adelson JD, Bourne RRAA, Briant PS, Flaxman SR, Taylor HRBB, Jonas JB, et al. Causes of blindness and vision impairment in 2020 and trends over 30 years, and prevalence of avoidable blindness in relation to VISION 2020: the Right to Sight: an analysis for the Global Burden of Disease Study. Lancet Glob Health. 2021;9: e144-60.

2. Stein JD, Khawaja AP, Weizer JS. Glaucoma in adults-screening, diagnosis, and management: a review. JAMA. 2021;325:164-74.

3. McCann P, Hogg R, Wright DM, Pose-Bazarra S, Chakravarthy U, Peto T, et al. Glaucoma in the Northern Ireland Cohort for the Longitudinal Study of Ageing (NICOLA): cohort profile, prevalence, awareness and associations. $\mathrm{Br} J$ Ophthalmol. 2020;104:1492-9.

4. UK National Screening Committee. Screening for Glaucoma: External review against programme appraisal criteria for the UK National Screening Committee. 2019. https://legacyscreening.phe.org.uk/glaucoma.

5. UK National Screening Committee. UK NSC: evidence review process. 2017. https://www.gov.uk/government/publications/uk-nsc-evidence-review-process/ uk-nsc-evidence-review-process. Accessed 16 April 2021.

6. Boland MV, Gupta P, Ko F, Zhao D, Guallar E, Friedman DS. Evaluation of frequency-doubling technology perimetry as a means of screening for glaucoma and other eye diseases using the national health and nutrition examination survey. JAMA Ophthalmol. 2016;134:57-62. 
7. Wahl J, Barleon L, Morfeld P, Lichtmeß A, Haas-Brähler S, Pfeiffer N. The Evonik-Mainz Eye Care-Study (EMECS): development of an expert system for glaucoma risk detection in a working population. PLoS ONE. 2016;11:1-24.

8. Zhao D, Guallar E, Gajwani P, Swenor B, Crews J, Saaddine J, et al. Optimizing glaucoma screening in high-risk population: design and 1-year findings of the screening to prevent (SToP) glaucoma study. Am J Ophthalmol. 2017;180:18-28.

9. Song YJ, Kim YW, Park KH, Kim YK, Choi HJ, Jeoung JW. Comparison of glaucoma patients referred by glaucoma screening versus referral from primary eye clinic. PLoS ONE. 2019;14:1-9.

10. Dabasia PL, Fidalgo BR, Edgar DF, Garway-Heath DF, Lawrenson JG. Diagnostic accuracy of technologies for glaucoma case-finding in a community setting. Ophthalmology. 2015;122:2407-15.

11. Hark LA, Myers JS, Ines A, Jiang A, Rahmatnejad K, Zhan T, et al. Philadelphia Telemedicine Glaucoma Detection and Follow-up Study: confirmation between eye screening and comprehensive eye examination diagnoses. $\mathrm{Br} J$ Ophthalmol. 2019;103:1820-6.

12. Hatt $S$, Wormald R, Burr J. Screening for prevention of optic nerve damage due to chronic open angle glaucoma. Cochrane database Syst. Rev. 2006: CD006129. https://doi.org/10.1002/14651858.CD006129.pub2.

13. Ervin A-M, Boland MV, Myrowitz EH, Prince J, Hawkins B, Vollenweider D, et al. Screening for glaucoma: comparative effectiveness. US: Agency for Healthcare Research and Quality; 2012.

14. Moyer VA \& U.S. Preventive Services Task Force. Screening for glaucoma: U.S. preventive services task force recommendation statement. Ann Intern Med. 2013;159:484-9.

15. Burr J, Hernández R, Ramsay $C$, Prior $M$, Campbell $S$, Azuara-Blanco A, et al. Is it worthwhile to conduct a randomized controlled trial of glaucoma screening in the United Kingdom? J Health Serv Res Policy. 2014;19:42-51.

16. Anton A, Fallon M, Cots F, Sebastian MA, Morilla-Grasa A, Mojal S, et al. Cost and detection rate of glaucoma screening with imaging devices in a primary care center. Clin Ophthalmol. 2017;11:337-46.

17. National Institute for Health and Care Excellence. Glaucoma: diagnosis and management NICE guideline [NG81]. 2017. https://www.nice.org.uk/guidance/ng81. Accessed 17 Feb 2021

18. Burr JM, Mowatt G, Hernández R, Siddiqui MAR, Cook J, Lourenco $T$, et al. The clinical effectiveness and cost-effectiveness of screening for open angle glaucoma: a systematic review and economic evaluation. Health Technol Assess. 2007;11:1-190.

19. Azuara-Blanco A, Burr J, Thomas R, Maclennan G, McPherson S. The accuracy of accredited glaucoma optometrists in the diagnosis and treatment recommendation for glaucoma. Br J Ophthalmol. 2007;91:1639-43.

20. Torjesen I. Glaucoma report: patients' sight is put at risk by treatment delays. BMJ. 2020;368:m103.

21. Healthcare Safety Investigation Branch. Lack of timely monitoring of patients with glaucoma. Healthcare Safety Investigation I2019/001. 2020:1-64. https://www.hsib. org.uk/documents/199/hsib_report_lack_timely_monitoring_patients_glaucoma.pdf.

22. Wolfs RC, Klaver CC, Ramrattan RS, van Duijn CM, Hofman A, de Jong PT. Genetic risk of primary open-angle glaucoma. Population-based familial aggregation study. Arch Ophthalmol. 1998;116:1640-5.

23. Choquet H, Wiggs JL, Khawaja AP. Clinical implications of recent advances in primary open-angle glaucoma genetics. Eye. 2020;34:29-39.

24. Khawaja AP, Cooke Bailey JN, Wareham NJ, Scott RA, Simcoe M, Igo RP, et al. Genome-wide analyses identify 68 new loci associated with intraocular pressure and improve risk prediction for primary open-angle glaucoma. Nat Genet. 2018;50:778-82.

25. Craig JE, Han X, Qassim A, Hassall M, Cooke Bailey JN, Kinzy TG, et al. Multitrait analysis of glaucoma identifies new risk loci and enables polygenic prediction of disease susceptibility and progression. Nat Genet. 2020;52:160-6.

26. Neustaeter A, Nolte I, Snieder H, Jansonius NM. Genetic pre-screening for glaucoma in population-based epidemiology: protocol for a double-blind prospective screening study within Lifelines (EyeLife). BMC Ophthalmol. 2021;21:1-8.

27. Hauser MA, Allingham RR, Aung T, Van Der Heide CJ, Taylor KD, Rotter Jl, et al. Association of genetic variants with primary open-angle glaucoma among individuals with african ancestry. JAMA. 2019;322:1682-91.

28. Gharahkhani P, Jorgenson E, Hysi P, Khawaja AP, Pendergrass S, Han X, et al. Genomewide meta-analysis identifies 127 open-angle glaucoma loci with consistent effect across ancestries. Nat Commun. 2021;12:1258.

29. Mayro EL, Wang M, Elze T, Pasquale LR. The impact of artificial intelligence in the diagnosis and management of glaucoma. Eye. 2020;34:1-11.

30. Phene S, Dunn RC, Hammel N, Liu Y, Krause J, Kitade N, et al. Deep learning and glaucoma specialists: the relative importance of optic disc features to predict glaucoma referral in fundus photographs. Ophthalmology. 2019;126:1627-39.
31. NHS Services. Free NHS eye tests and optical vouchers. 2020. https://www.nhs.uk/ nhs-services/opticians/free-nhs-eye-tests-and-optical-vouchers/. Accessed 22 March 2021.

32. NHS Inform. Your entitlements to NHS ophthalmic services. https://www. nhsinform.scot/care-support-and-rights/nhs-services/ophthalmics/yourentitlements-to-nhs-ophthalmic-services. Accessed 22 March 2021.

33. NHS Wales. Eye Health Examination Wales (EHEW) Service: A clinical manual with protocols for optometrists, ophthalmic medical practitioners (OMPs) and contractors |Version 18. 2018:1-66. http://www.eyecare.wales.nhs.uk/sitesplus/documents/1082/ EHEWManualrefreshv182018FINALFINAL.pdf. Accessed 22 March 2021.

34. Health and Social Care Board. Ophthalmic services. 2021. http://www.hscboard. hscni.net/our-work/integrated-care/ophthalmic-services/. Accessed 22 March 2021.

35. Khawaja AP, Sherratt M-A, Sparrow JM. Royal College of Ophthalmologists Glaucoma Commissioning Guidance Development Group. The Royal College of Ophthalmologists' Glaucoma Commissioning Guidance: executive summary. Eye (Lond). 2017:1-5. https://doi.org/10.1038/eye.2017.11.

36. NHS England. What are integrated care systems? https://www.england.nhs.uk/ integratedcare/what-is-integrated-care/. Accessed 22 March 2021.

37. Vaahtoranta-Lehtonen $H$, Tuulonen A, Aronen $P$, Sintonen $H$, Suoranta $L$, Kovanen $\mathrm{N}$, et al. Cost effectiveness and cost utility of an organized screening programme for glaucoma. Acta Ophthalmol Scand. 2007;85:508-18.

38. Tang J, Liang Y, O'Neill C, Kee F, Jiang J, Congdon N. Cost-effectiveness and costutility of population-based glaucoma screening in China: a decision-analytic Markov model. Lancet Glob Health. 2019;7:e968-e978.

39. Rahman F, Zekite A, Bunce $C$, Jayaram $H$, Flanagan D. Recent trends in vision impairment certifications in England and Wales. Eye. 2020;34:1271-8.

\section{ACKNOWLEDGEMENTS}

APK is funded by a UKRI Future Leaders Fellowship and an Alcon Research Institute Young Investigator Award.

\section{AUTHOR CONTRIBUTIONS}

$\mathrm{SH}$ and APK conceived and drafted the manuscript. PD, JMB and PH critically appraised and revised the manuscript. All authors approved the final version.

\section{COMPETING INTERESTS}

APK has acted as a consultant to Aerie, Allergan, Google Health, Novartis, Reichert, Santen and Thea.

\section{ADDITIONAL INFORMATION}

Correspondence and requests for materials should be addressed to A.P.K.

Reprints and permission information is available at http://www.nature.com/ reprints

Publisher's note Springer Nature remains neutral with regard to jurisdictional claims in published maps and institutional affiliations.

\begin{abstract}
Open Access This article is licensed under a Creative Commons cc) Attribution 4.0 International License, which permits use, sharing, appropriate credit to the original author(s) and the source, provide a link to the Creative Commons license, and indicate if changes were made. The images or other third party material in this article are included in the article's Creative Commons license, unless indicated otherwise in a credit line to the material. If material is not included in the article's Creative Commons license and your intended use is not permitted by statutory regulation or exceeds the permitted use, you will need to obtain permission directly from the copyright holder. To view a copy of this license, visit http://creativecommons. org/licenses/by/4.0/.
\end{abstract}

(c) The Author(s) 2021 